\title{
HIGHER DEGREES OF DISTRIBUTIVITY IN LATTICES OF CONTINUOUS FUNCTIONS $\left({ }^{1}\right)$
}

\author{
BY \\ ELLIOT CARL WEINBERG
}

This work is concerned with lattice distributivity properties expressed by lattice identities with infinitely many variables. A distributive lattice may be described as one in which the following equation and its lattice dual hold identically whenever $J$ and $I$ are finite index sets:

$$
\Pi_{i \in I} \Sigma_{j \in J} a_{i, j}=\Sigma_{\phi \in J^{I}} \Pi_{i \in I} a_{i, \phi(i)} .
$$

This suggests calling a lattice $(\alpha, \beta)$-distributive when the above equations are valid for index sets $I$ and $J$ of cardinality $\alpha$ and $\beta$ respectively.

The first appearance of the notion of $(\alpha, \beta)$-distributivity was in a problem concerning Boolean algebras posed by Erdös and Tarski [3]. More recently, a number of papers dealing with $(\alpha, \beta)$-distributive Boolean algebras have appeared. These include works by R. S. Pierce [8; 9], D. Scott [11], E. C. Smith, Jr. [13], and the latter author together with A. Tarski [14].

Among Pierce's results is the following theorem: if $\alpha$ is an infinite cardinal and $A$ is an $\alpha$-complete Boolean algebra, then $A$ is $(\alpha, \alpha)$-distributive if and only if the lattice of all continuous real-valued functions on the Stone space of $A$ is $(\alpha, \alpha)$-distributive. Our work is motivated by this theorem. Among the results obtained is a generalization of Pierce's theorem which omits the hypotheses that $A$ is $\alpha$-complete and $\alpha=\beta$.

The major step requires a translation of the statement that the lattice $C(X)$ of all real-valued continuous functions on the topological space $X$ is $(\alpha, \beta)$-distributive into topological properties of the underlying space. Using this translation we then prove that $C(X)$ is $(\alpha, \alpha)$-distributive whenever it is $(\alpha, 2)$-distributive, a result already known for Boolean algebras. The validity of this implication is apparently unsettled for the more general case of an arbitrary lattice or even for archimedean lattice-ordered groups.

In the last section we present some examples and generalize another theorem of Pierce: if $C(X)$ is $(\alpha, \alpha)$-distributive when $\alpha$ is the first infinite cardinal, then every continuous real-valued function defined on $X$ is locally constant

Received by the editors September 11, 1961.

(1) This work is a portion of a doctoral dissertation submitted to the faculty of Purdue University in January, 1961 and was supported by the National Science Foundation and the Office of Naval Research (Contract No. Nonr-1100 (12)). The author is very much indebted to Professor Melvin Henriksen for his advice during the period of preparation of the original thesis. 
on a dense set. In another paper we will prove that if $C(X)$ is $(\alpha, \alpha)$-distributive, then it may be embedded as a regular sublattice of a $C(Y)$ which is $\alpha$-complete and $(\alpha, \alpha)$-distributive.

1. Notation and terminology. Each cardinal will be identified with the smallest ordinal of that power. For example, the first infinite cardinal will be denoted $\omega_{0}$. Under this convention each cardinal $\alpha$ is a totally-ordered set. Moreover, $i \in \alpha$ is equivalent to $i<\alpha$. If $\alpha$ and $\beta$ are cardinals, the symbols $\alpha^{\beta}$ and $\alpha \times \beta$ will denote, respectively, the set of all functions from the set $\beta$ into the set $\alpha$ and the Cartesian product of the sets $\alpha$ and $\beta$. An $\alpha$-sequence is a set indexed by the cardinal $\alpha$. The set of positive integers will be denoted by $N$.

All topological spaces considered in this paper are Hausdorff and completely regular. Recall that a Hausdorff space is completely regular provided that for each closed set $H$ and each point $p$ not in $H$, there exists a continuous function from $X$ into the space $R$ of real numbers which assumes the values 0 at $p$ and 1 at each point of $H$. A subset $Z$ of the space $X$ is called a zeroset if there exists a real-valued continuous function $f$ on $X$ such that $Z=\{x: f(x)=0\}$. A subset of $X$ is called a cozeroset if it is the complement of a zeroset. Each cozeroset $U$ is the positive set of a continuous real-valued function $f$; that is, $U=\operatorname{pos} f=\{x: f(x)>0\}$. Observe that a Hausdorff space $X$ is completely regular precisely when the lattice $U(X)$ of all cozerosets of $X$ is a base for the topology of $X$. If the Boolean algebra $B(X)$ of all subsets of $X$ which are both open and closed in $X$ forms a base for the topology of $X$, then we say that $X$ is zerodimensional.

The closure (respectively interior) of a set $H$ in the space $X$ will be denoted $\mathrm{cl} H$ or $\mathrm{cl}_{X} H$ (respectively int $H$ or int ${ }_{X} H$ ). If $f$ is a function defined on $X$, then $f \mid H$ will denote the restriction of $f$ to $H$. For general topological background the reader should refer to [4].

Let $L$ be a lattice. Least upper bounds (greatest lower bounds) in $L$ will be denoted by $\Sigma$ or $\vee(\Pi$ or $\wedge)$. The lattice $L$ is called $\alpha$-complete if each bounded $\alpha$-sequence of elements of $L$ has a least upper bound and a greatest lower bound. A lattice homomorphism $\bar{t}$ of one lattice into another is called $\alpha$-complete if $\Sigma_{i<\alpha} a_{i}=a$ implies $\Sigma_{i<\alpha} \bar{t} a_{i}=\bar{t} a$. A lattice (or lattice homomorphism) is called complete if it is $\alpha$-complete for every cardinal $\alpha$. If $L$ is a sublattice of $M$, then it is called a regular sublattice if the injection map is a complete isomorphism.

An $l$-group $G$ is a group with a relation $\leqq$ such that $(G, \leqq)$ is a lattice in which group translations are lattice automorphisms: for each $g \in G$, $(x-g) \vee(y-g)=(x \vee y)-g$ for all $x, y \in G$. Let 0 denote the identity of the group. The set $\{g \in G ; 0 \leqq g\}$ of positive elements of $G$ will be denoted $G^{+}$. An element $u \in G^{+}$is called a weak order unit if $u \wedge x=0$ implies $x=0$. If $g \in G$, then $|g|=g \bigvee(-g)$. A subgroup $H$ of $G$ is called convex if $h \in H$ and $0 \leqq g \leqq h$ implies $g \in H$. A subgroup is called absolutely convex if it is a 
convex sub-l-group. An $l$-group is called archimedean if, for each $g>0$, the set $\{n g: n=1,2, \cdots\}$ has no upper bound in $G$.

By an $l$-algebra $A$, we mean an $l$-group which is an algebra over the real field $R$ in which the product of positive elements is positive and the product of a positive element with a positive real number is positive. We will be concerned with a special class of $l$-algebras called $\Phi$-algebras. A $\Phi$-algebra is an archimedean $l$-algebra which has a multiplicative identity 1 that is a weak order unit. If $A$ is a $\Phi$-algebra, then the set $A^{*}=\{a \in A:|a| \leqq r \cdot 1$ for some $r \in R\}$ of bounded elements of $A$ is also a $\Phi$-algebra. The $\Phi$-algebra $A$ is called bounded if $A=A^{*}$. An $l$-ideal of a $\Phi$-algebra is a ring ideal which is absolutely convex.

The algebra $C(X)$ of all continuous real-valued functions on the topological space $X$ with the operations defined pointwise is clearly a $\Phi$-algebra. When discussing $C(X)$ we will use the same symbol to denote the real number $r$ and the constant function which assumes only the value $r$. A subset $A$ of $C(X)$ is uniformly dense if for each $f \in C(X)$ and for each $n \in N$, there exists $a \in A$ such that $|f-a| \leqq 1 / n$.

1.1. Proposition. If $A$ is a uniformly dense sub- $\Phi$-algebra of $C(X)$, then $A$ is a regular sublattice of $C(X)$.

Proof. Suppose that $a, a_{i} \in A(i \in I), f \in C(X)$, and $a_{i} \leqq f<a$ for all $i \in I$. There exists $n \in N$ such that $a \wedge(f+1 / n)<a$. We can find $b \in A$ such that $f-1 / 2 n \leqq b \leqq f+1 / 2 n$. Then $f \leqq b+1 / 2 n \leqq f+1 / n$, so $a_{i} \leqq(b+1 / 2 n) \wedge a<a$ for all $i \in I$. Since $(b+1 / 2 n) \wedge a \in A, a$ is not the least upper bound in $A$ of the set $\left\{a_{i}: i \in I\right\}$.

If the $\Phi$-algebra $A$ is bounded, it can actually be represented as a uniformly dense sub- $\Phi$-algebra of $C(X)$ for some compact space $X$. The space in question has for its points the family $M(A)$ of maximal $l$-ideals of $A$. A basic open set is of the form $\{\mathfrak{T} \in M(A): f \notin \mathfrak{N}\}$ for some $f \in A$. For a more thorough discussion and a proof of the following proposition, see [6].

1.2. Proposition. Let $A$ be a $\Phi$-algebra. The space $M(A)$ of maximal $l$ ideals of $A$ is a compact Hausdorff space. Moreover, $A^{*}$ is isomorphic to a uniformly dense sub- $\Phi$-algebra of $C(M(A))$.

In case $A=C(X), M(A)$ is the familiar Stone-Cech compactification of $X$ (here denoted by $\beta X$ ), and $C(M(A))$ is isomorphic to $C^{*}(X)$.

Brief use of the following notions will be made in $\S \S 3$ and 4 . A complete discussion and a proof of 1.4 will appear elsewhere. Let $X$ and $Y$ be topological spaces, and let $t$ be a continuous map of $X$ into $Y$. Let $\bar{t}$ be the homomorphism of $C(Y)$ into $C(X)$ induced by $t:(\bar{t} f)(x)=f(t(x))$.

1.3. Definition. Let $\alpha$ be an infinite cardinal. The map $t$ is $\alpha$-complete if int $\bigcap_{i<\alpha} t^{-1}\left(U_{i}\right)$ is empty whenever $\left\{U_{i}: i<\alpha\right\}$ is an $\alpha$-sequence of open sets in 
$Y$ such that int $\bigcap_{i<\alpha} U_{i}$ is empty. The map $t$ is complete if it is $\alpha$-complete for every cardinal $\alpha$. (For example, open continuous maps are complete.)

1.4. Proposition. Let $t$ be a continuous function from $X$ into $Y$. If $t$ is $\alpha$-complete, then $\bar{t}$ is $\alpha$-complete.

2. $(\alpha, \beta)$-distributivity. 2.1. Definition. A lattice $L$ is $(\alpha, \beta)$-distributive if both (a) and (b) are satisfied:

$$
\text { (a) } \Pi_{i<\alpha} \Sigma_{j<\beta} f_{i, j}=\Sigma_{\phi \in \beta^{\alpha}} \Pi_{i<\alpha} f_{i, \phi(i)}
$$

whenever $\left\{f_{i, j}: i<\alpha, j<\beta\right\} \subset L$ and all the indicated suprema and infima exist.

(b) $\Sigma_{i<\alpha} \Pi_{j<\beta} f_{i, j}=\Pi_{\phi \in \beta^{\alpha}} \Sigma_{i<\alpha} f_{i, \phi(i)}$

whenever $\left\{f_{i, j}: i<\alpha, j<\beta\right\} \subset L$ and all the indicated suprema and infima exist.

2.2. REmarks. (a) Every regular sublattice of an $(\alpha, \beta)$-distributive lattice is $(\alpha, \beta)$-distributive. (b) A lattice is distributive iff it is $(m, n)$-distributive for all finite cardinals $m$ and $n$. (c) If $L$ is $(\alpha, \beta)$-distributive, $1<\alpha^{\prime}<\alpha$, and $1<\beta^{\prime}<\beta$, then $L$ is $\left(\alpha^{\prime}, \beta^{\prime}\right)$-distributive. (d) In an $l$-group, 2.1(a) is true iff 2.1(b) is true. (e) If $\left\{f_{i, j}: i<\alpha, j<\beta\right\}$ is contained in the lattice $L$, then $\Pi_{i<\alpha} \Sigma_{j<\beta} f_{i, j} \geqq \Sigma_{\phi \in \beta^{\alpha}} \Pi_{i<\alpha} f_{i, \phi(i)}$ whenever all the indicated suprema and infima exist.

2.3. Definition. A lattice $L$ is infinitely distributive if $a, a_{i} \in L(i \in I)$ and $\Sigma_{i \in I} a_{i}$ exists implies $\Sigma_{i \in I} a \wedge a_{i}$ exists and equals $a \wedge \Sigma_{i \in I} a_{i}$.

2.4. Proposition. Every l-group is infinitely distributive. (See [1, p. 231].)

2.5. Corollary. Let $G$ be an l-group. For each finite $n$ and for each $\alpha, G$ is $(n, \alpha)$-distributive.

2.6. THEOREM. In any $l$-group $G$, the following conditions are equivalent:

(a) If $\left\{f_{i, j}: i<\alpha, j<\beta\right\}$ is a subset of $G$ for which $\Sigma_{j<\beta} f_{i, j}$ for all $i<\alpha$, $\Pi_{i<\alpha} \Sigma_{j<\beta} f_{i, j}$, and $\Pi_{i<\alpha} f_{i, \phi(i)}$ for each $\phi \in \beta^{\alpha}$ all exist, then $\Sigma_{\phi \in \beta^{\alpha}} \Pi_{i<\alpha} f_{i, \phi(i)}$ exists and equals $\Pi_{i<\alpha} \Sigma_{j<\beta} f_{i, j}$.

(b) The l-group $G$ is $(\alpha, \beta)$-distributive.

(c) If $\left\{f_{i, j}: i<\alpha, j<\beta\right\}$ is a subset of $G^{+}$for which there exists $f>0$ such that $\Sigma_{j<\beta} f_{i, j}=f$ for each $i<\alpha$, then there exists $\phi \in \beta^{\alpha}$ such that $\Pi_{i<\alpha} f_{i, \phi(i)}=0$ is false.

Proof. It is clear that (a) implies (b), which implies (c). Let $\left\{f_{i, j}: i<\alpha, j<\beta\right\}$ be a family of elements as described in (a). Let $f=\Pi_{i<\alpha} \Sigma_{j<\beta} f_{i, j}$. If $f$ is not equal to $\Sigma_{\phi \in \beta^{\alpha}} \Pi_{i<\alpha} f_{i, \phi(i)}$, then there exists $g$ in $G$ such that $\Pi_{i<\alpha} f_{i, \phi(i)} \leqq g<f$ for all $\phi \in \beta^{\alpha}$. Let $h_{i, j}=\left(f_{i, j} \wedge f\right)-g$ for each $i<\alpha, j<\beta$. Applying the infinite distributive law (2.4) and the fact that group translations are lattice automorphisms, we can calculate:

and

$$
\Sigma_{j<\beta} h_{i, j}=\left(f \wedge \Sigma_{j<\beta} f_{i, j}\right)-g=f-g>0 \quad \text { for all } i<\alpha,
$$




$$
\begin{aligned}
\Pi_{i<\alpha} h_{i, \phi(i)} & =\left(f \wedge \Pi_{i<\alpha} f_{i, \phi(i)}\right)-g \\
& =\left(\Pi_{i<\alpha} f_{i, \phi(i)}\right)-g \leqq 0 \quad \text { for all } \phi \in \beta^{\alpha} .
\end{aligned}
$$

Hence,

$$
\Sigma_{j<\beta}\left(h_{i, j} \bigvee 0\right)=f-g>0 \quad \text { for all } i<\alpha,
$$

while

$$
\Pi_{i<\alpha}\left(h_{i, \phi(i)} \vee 0\right)=0 \vee \Pi_{i<\alpha} h_{i, \phi(i)}=0 \quad \text { for all } \phi \in \beta^{\alpha} .
$$

Thus we see that (c) is false when (a) is false.

The equivalences of Theorem 2.6 were established for Boolean algebras by D. Scott [14]. Condition (c) is the easiest to verify and will be referred to frequently. We will have occasion to refer to the following proposition as well. For a proof see [8].

2.7. Proposition. In a Boolean algebra $A$, the following conditions are equivalent.

(a) The Boolean algebra $A$ is $(\alpha, \beta)$-distributive.

(b) If $\left\{a_{i, j}: i<\alpha, j<\beta\right\} \subset A$ and $\Sigma_{j<\beta} a_{i, j}=1$ for each $i<\alpha$, then there is a subset $S$ of $A$ such that $\Sigma_{s \in S} s=1$ and for each $s \in S$ there exists $\phi \in \beta^{\alpha}$ such that $s \leqq a_{i, \phi(i)}$ for all $i<\alpha$.

2.8. Proposition. If the l-group $G$ is $(\alpha, 2)$-distributive, then it is $(\alpha, n)$ distributive for all finite $n$.

Proof. The proof is by induction. Suppose that $G$ is $(\alpha, n)$-distributive for some finite $n \geqq 2$. Let $f, f_{i, j}(i<\alpha, j<n+1)$ be members of $G^{+}$satisfying $\Sigma_{j<n+1} f_{i, j}=f>0$ for all $i<\alpha$. Let

$$
\begin{aligned}
& g_{i, 0}=f_{i, n}, \text { and let } \\
& g_{i, 1}=\Sigma_{j<n} f_{i, j} .
\end{aligned}
$$

By Theorem 2.6, there exist $\phi \in 2^{\alpha}$ and $g \in G$ such that $0<g \leqq g_{i, \phi(i)}$. For each $j<n$, let

$$
\begin{aligned}
h_{i, j} & =f_{i, n} \wedge g=g \text { if } \phi(i)=0, \\
& =f_{i, j} \wedge g \text { if } \phi(i)=1 .
\end{aligned}
$$

For each $i<\alpha, \Sigma_{j<n} h_{i, j}=g>0$. By the induction hypothesis there exist $\psi \in n^{\alpha}$ and $h \in G$ satisfying $0<h \leqq h_{i, \psi(i)}$ for each $i<\alpha$. Define $\theta \in(n+1)^{\alpha}$ by $\theta(i)$ $=\psi(i)$ if $\phi(i)=1$, and $\theta(i)=n$ if $\phi(i)=0$. We now have $0<h \leqq h_{i, \psi(i)} \leqq f_{i, \theta(i)}$ for all $i<\alpha$. Thus $G$ is $(\alpha, n+1)$-distributive.

2.9. Proposition. Let $G$ be an l-group with a weak order unit $u$, and let $H$ be a regular, convex, sub-l-group of $G$ which contains $u$. Then $H$ is $(\alpha, \beta)$-distributive if and only if $G$ is $(\alpha, \beta)$-distributive.

Proof. If $\left\{f_{i, j}: i<\alpha, j<\beta\right\}$ is a family of members of $G^{+}$such that $\Sigma_{j<\beta} f_{i, j}$ 
$=f>0$ for each $i<\alpha$, although $\Pi_{i<\alpha} f_{i, \phi(i)}=0$ for all $\phi \in \beta^{\alpha}$, then $\Sigma_{j<\beta}\left(f_{i, j} \wedge u\right)$ $=f \wedge u>0$ for all $i<\alpha$, while $\Pi_{i<\alpha}\left(f_{i, \phi(i)} \wedge u\right)=0$ for all $\phi \in \beta^{\alpha}$. Thus $H$ fails to be $(\alpha, \beta)$-distributive along with $G$. The converse is a corollary to $2.2(\mathrm{a})$.

2.10. Corollary. (a) Let $A$ be a $\Phi$-algebra. Then $A$ is $(\alpha, \beta)$-distributive if and only if $A^{*}$ is $(\alpha, \beta)$-distributive.

(b) Let $X$ be a topological space. Then $C(X)$ is $(\alpha, \beta)$-distributive if and only if $C^{*}(X)$ is $(\alpha, \beta)$-distributive.

2.11. Theorem. Let $A$ be a $\Phi$-algebra and let $M(A)$ be the space of maximal l-ideals of $A$. Then $A$ is $(\alpha, \beta)$-distributive if and only if $C(M(A))$ is $(\alpha, \beta)$ distributive.

Proof. Recall that $A^{*}$ is isomorphic to a uniformly dense sub- $\Phi$-algebra $B$ of $C(M(A))$ (1.2); moreover, $B$ is a regular sublattice of $C(M(A))$ (1.1). Thus $B, A^{*}$, and, by $2.10(\mathrm{a}), A$ are $(\alpha, \beta)$-distributive whenever $C(M(A))$ is.

Conversely, suppose that $A$, and hence $B$, is $(\alpha, \beta)$-distributive. Let $f$, $f_{i, j}(i<\alpha, j<\beta)$ be a family of members of $C(M(A))^{+}$satisfying $\Sigma_{j<\beta} f_{i, j}$ $=f>0$ for each $i<\alpha$. Since $B$ is uniformly dense in $C(M(A))$, there exists $b \in B$ such that $0<b<f$. Then $\Sigma_{j<\beta}\left(b \wedge f_{i, j}\right)=b \wedge f=b$ for all $i<\alpha$. There is a positive integer $n$ such that $c=0 \vee(b-1 / n)>0$. For each pair $i<\alpha, j<\beta$, we can find $b_{i, j} \in B$ satisfying $0 \vee\left[\left(f_{i, j} \wedge b\right)-1 / n\right] \leqq b_{i, j} \leqq f_{i, j} \wedge b \wedge c$. Observe that $\Sigma_{j<\beta}\left(0 \vee\left[\left(f_{i, j} \wedge b\right)-1 / n\right]\right)=c$. Hence, if, for fixed $i<\alpha, d \geqq b_{i, j}$ for all $j<\beta$, then $d \geqq c$; so $\Sigma_{j<\beta} b_{i, j}=c$ for all $i<\alpha$. Since $B$ is $(\alpha, \beta)$-distributive, there exist $\phi \in \beta^{\alpha}$ and $d \in B$ such that $0<d \leqq b_{i, \phi(i)}$ for all $i<\alpha$. We have $d \leqq f_{i, \phi(i)}$, so $C(M(A))$ is $(\alpha, \beta)$-distributive.

The significance of 2.11 is clear. If we want to establish that a result on infinite distributive laws holds in all $\Phi$-algebras, we need only show that it holds for $C(X)$ whenever $X$ is compact.

3. Lattices of continuous functions. In this section we begin the discussion of $(\alpha, \beta)$-distributivity in the lattice $C(X)$ of all continuous real-valued functions on a topological space $X$. Our major goal is to achieve a topological characterization of this lattice property in terms of topological properties of the underlying space. The symbol $X$ will always denote a completely regular space.

3.1. Proposition. If $C(X)$ is $(\alpha, \beta)$-distributive, and $U$ is an open subset of $X$, then $C(U)$ is $(\alpha, \beta)$-distributive.

Proof. Suppose that $C(U)$ is not $(\alpha, \beta)$-distributive. Then we can find $f, f_{i, j} \in C(U)^{+}(i<\alpha, j<\beta)$ such that $\Sigma_{j<\beta} f_{i, j}=f>0$ for each $i<\alpha$, while $\Pi_{i<\alpha} f_{i, \phi(i)}=0$ for all $\phi \in \beta^{\alpha}$. There exists $g \in C(X)^{+}$such that $g$ vanishes on a neighborhood $V$ of $X-U$ and agrees with $f$ at some point in the positive set of $f$. For each pair $i<\alpha, j<\beta$, define $h_{i, j}$ to agree with $g$ on $X-U$ and with $f_{i, j} \wedge g$ on $U$. Define $h$ to agree with $g$ on $X-U$ and with $f \wedge g$ on $U$. Each of 
the new functions is continuous on both $V$ and $U$; hence they are all continuous on $X$.

Let $\phi \in \beta^{\alpha}$. If $0 \leqq k \in C(X)$ and $k \leqq h_{i, \phi(i)}$ for all $i<\alpha$, then $k \mid U \leqq f_{i, \phi(i)}$ for all $i<\alpha$, so $k \mid U=0$. Since each $h_{i, j}$ is zero on $X-U$, we have $k=0$, i.e., $\Pi_{i<\alpha} h_{i, \phi(i)}=0$ for each $\phi \in \beta^{\alpha}$.

Certainly $h_{i, j} \leqq h$ for each $i<\alpha, j<\beta$. Suppose, for fixed $i$, that $k \in C(X)^{+}$ and $h_{i, j} \leqq k$ for each $j<\beta$. Then $f_{i, j} \wedge(g \mid U)=h_{i, j}|U \leqq k| U$. But $h$ is zero outside of $U$, so $h \leqq k$. Hence $h=\Sigma_{j<\beta} h_{i, j}>0$ for each $i<\alpha$. This completes the proof that $C(X)$ is not $(\alpha, \beta)$-distributive whenever $C(U)$ is not $(\alpha, \beta)$-distributive.

3.2. Corollary. If $C(X)$ is $(\alpha, \beta)$-distributive, $Y$ is contained in $X$, and int $Y$ is dense in $Y$, then $C(Y)$ is $(\alpha, \beta)$-distributive.

Proof. By $1.4, C(Y)$ may be embedded as a regular sublattice of $C$ (int $Y$ ), which is $(\alpha, \beta)$-distributive by Proposition 3.1.

3.3. Corollary. If $X=\mathrm{cl} \bigcup_{i \in I} U_{i}$, where $U_{i}$ is open in $X$ and $C\left(U_{i}\right)$ is $(\alpha, \beta)$-distributive for each $i \in I$, then $C(X)$ is $(\alpha, \beta)$-distributive.

Proof. Suppose that this is not the case. Then for some $f, f_{i, j} \in C(X)$ $(i<\alpha, j<\beta), \Sigma_{j<\beta} f_{i, j}=f>0$ for each $i<\alpha$, although $\Pi_{i<\alpha} f_{i, \phi(i)}=0$ for all $\phi \in \beta^{\alpha}$. There exists $k \in I$ such that $V=U_{k} \cap \operatorname{pos} f$ is not empty. By 1.4, the map $\bar{e}: C(X) \rightarrow C(V)$ induced by the injection map $e$ of $V$ into $X$ is a complete homomorphism. Hence $\Sigma_{j<\beta}\left(f_{i, j} \mid V\right)=f \mid V>0$ for all $i<\alpha$, while $\Pi_{i<\alpha}\left(f_{i, \phi(i)} \mid V\right)$ $=0$ for all $\phi \in \beta^{\alpha}$. This tells us that $C(V)$ and, by 3.1, $C\left(U_{k}\right)$ are not $(\alpha, \beta)$ distributive.

3.4. Definition. An almost-cover of a topological space $X$ is a family of cozerosets of $X$ whose union is dense in $X$. An almost- $\beta$-cover is an almost-cover of cardinality $\beta$.

Pierce [8] calls a cover of a Boolean algebra any family of elements whose least upper bound is 1 . A $\beta$-cover is a cover of cardinality $\beta$. Proposition 2.7 , expressed in these terms, says that the following condition is necessary and sufficient for the Boolean algebra $A$ to be $(\alpha, \beta)$-distributive: if $a_{i}$ is a $\beta$-cover of $A$ for each $i<\alpha$, then there is a cover of $A$ which refines each $\alpha_{i}$. (Recall that a family $S$ of elements of a partially-ordered set refines the family $T$ if for each $s$ in $S$ there exists $t$ in $T$ such that $s \leqq t$.) We will prove a similar theorem for $C(X)$ by using the notion of almost-cover. The topological characterization of $(\alpha, \beta)$-distributivity which is obtained can be used to generalize results concerning Boolean algebras and in some places to simplify the proofs. We particularly call the reader's attention to Theorem 3.9 which was first proved for compact $X$ with $C(X) \alpha$-complete and $\alpha=\beta$ by Pierce [8] using the Stone-Weierstrass Theorem. The proof given below is set theoretic.

Perhaps we should point out the device used to replace the operation of complementation. Although the lattice $U(X)$ of cozerosets of $X$ is not a 
complemented lattice, for each $U \in U(X)$ there exists a sequence $\left\{U_{n}: n<\omega_{0}\right\}$ contained in $U(X)$ such that:

(a) $U \cup U_{n}=X$ for all $n<\omega_{0}$, and

(b) if $V \in U(X)$ and $V \subset U_{n}$ for all $n<\omega_{0}$, then $V \cap U$ is empty. This follows from the observation that $X-U$ is a zeroset and hence is the intersection of a countable family of cozerosets.

3.5. ThEOREM. The following conditions are equivalent.

(a) $C(X)$ is $(\alpha, \beta)$-distributive.

(b) If $\mathfrak{u}_{i}$ is an almost- $\beta$-cover of $X$ for each $i<\alpha$, then there exists an almostcover $\mathcal{u}$ of $X$ which refines each $\mathfrak{u}_{i}$.

Proof. It is clear that both statements are true for finite $\alpha$, so we will assume that $\alpha$ is infinite.

Suppose that $C(X)$ is $(\alpha, \beta)$-distributive, and let $\left\{U_{i, j}: j<\beta\right\}$ be an almost$\beta$-cover of $X$ for each $i<\alpha$. We will begin the proof by showing that there exists $\phi \in \beta^{\alpha}$ such that int $\bigcap_{i<\alpha} U_{i, \phi(i)}$ is not empty. Suppose first that $\beta \leqq \alpha$. Since $U_{i, j}$ is a cozeroset, for each pair $i<\alpha, j<\beta$, there exists a sequence $\left\{U_{i, j, k}: k<\omega_{0}\right\}$ in $U(X)$ such that $\bigcap_{k<\omega_{0}} U_{i, j, k}=X-U_{i, j}$. For each triplet $i<\alpha, j<\beta$, and $k<\omega_{0}$, let

$$
\begin{aligned}
& V_{i, j, k, 0}=U_{i, j}, \text { and let } \\
& V_{i, j, k, 1}=U_{i, j, k} .
\end{aligned}
$$

For $r=0,1$, pick $f_{i, j, k, r}$ in $C(X)^{+}$such that $\operatorname{pos} f_{i, j, k, r}=V_{i, j, k, r}$. Clearly $Z\left(f_{i, j, k, 0} \vee f_{i, j, k, 1}\right)$ is empty, so the multiplicative inverse $g_{i, j, k}$ of $\left(f_{i, j, k, 0} \vee f_{i, j, k, 1}\right)$ exists for each triplet $i<\alpha, j<\beta$, and $k<\omega_{0}$. Let $h_{i, j, k, r}$ $=\left(g_{i, j, k}\right)\left(f_{i, j, k, r}\right)$. Then $h_{i, j, k, 0} \bigvee h_{i, j, k, 1}=1$ for each triplet $i<\alpha, j<\beta, k<\omega_{0}$. The cardinality of $\alpha \times \beta \times \omega_{0}$ is $\alpha$, so, by the distributivity hypothesis, there exist $\phi \in 2^{\alpha \times \beta \times \omega_{0}}$ and $h \in C(X)$ such that $0<h \leqq h_{i, j, k, \phi(i, j, k)}$ for all $i<\alpha, j<\beta$, $k<\omega_{0}$. Now,

$$
\varnothing \neq \operatorname{pos} h \subset \bigcap_{i<\alpha, j<\beta, k<\omega_{0}} \operatorname{pos} h_{i, j, k, \phi(i, j, k)} .
$$

Fix $i<\alpha$. There must be $j=\psi(i)<\beta$ and $\lambda(i)<\omega_{0}$ such that $\phi(i, \psi(i), \lambda(i))=0$, for otherwise

$$
\begin{aligned}
\operatorname{pos} h & \subset \bigcap_{j<\beta, k<\omega_{0}} \operatorname{pos} h_{i, j, k, 1}=\bigcap_{j<\beta, k<\omega_{0}} \operatorname{pos} f_{i, j, k, 1} \\
& =\bigcap_{j<\beta, k<\omega_{0}} U_{i, j, k}=\bigcap_{j<\beta}\left(X-U_{i, j}\right),
\end{aligned}
$$

which has empty interior. We have $\varnothing \neq \operatorname{pos} h \subset \bigcap_{i<\alpha} U_{i, \psi(i)}$.

Now suppose that $\beta$ exceeds $\alpha$. For each pair $i<\alpha, j<\beta$, there exists a sequence of zerosets $\left\{Z_{i, j, k}: k<\omega_{0}\right\}$ such that $U_{i, j}=\bigcup_{k<\omega_{0}} Z_{i, j, k}$. For each $i<\alpha$, $j<\beta, k<\omega_{0}$, pick $f_{i, j, k}$ in $C(X)$ satisfying:

$$
\begin{aligned}
& 0 \leqq f_{i, j, k} \leqq 1, \\
& X-U_{i, j} \subset Z\left(f_{i, j, k}\right), \quad \text { and } \quad Z\left(1-f_{i, j, k}\right)=Z_{i, j, k} .
\end{aligned}
$$


Then, $\Sigma_{j<\beta, k<\omega_{0}} f_{i, j, k}=1$ for each $i<\alpha$ since

$$
\operatorname{cl} \bigcup_{j<\beta, k<\omega_{0}} Z_{i, j, k}=\operatorname{cl} \bigcup_{j<\beta} U_{i, j}=X .
$$

By the distributivity hypothesis, there exist functions $\psi \in \omega_{0}^{\alpha}, \phi \in \beta^{\alpha}$, and $g \in C(X)$ such that $0<g \leqq f_{i, \phi(i), \psi(i)}$ for each $i<\alpha$. Hence

$$
\varnothing \neq \operatorname{pos} g \subset \bigcap_{i<\alpha} \operatorname{pos} f_{i, \phi(i), \psi(i)} \subset \bigcap_{i<\alpha} U_{i, \phi(i)} .
$$

If $V$ is a nonempty open subset of $X$, then $\left\{V \cap U_{i, j}: j<\beta\right\}$ is an almost- $\beta$ cover of $V$ for each $i<\alpha$. By Proposition 3.1, $C(V)$ is $(\alpha, \beta)$-distributive, so the result of the previous paragraphs tells us that there exists $\phi \in \beta^{\alpha}$ such that int $_{V} \bigcap_{i<\alpha}\left(V \cap U_{i, \phi(i)}\right)=V \cap \operatorname{int}_{X} \bigcap_{i<\alpha} U_{i, \phi(i)} \neq \varnothing$. Hence, $U_{\phi \in \beta^{\alpha}}$ int $\bigcap_{i<\alpha} U_{i, \phi(i)}$ is dense in $X$. If $\digamma_{\phi}$ is a family of cozerosets of $X$ such that $U \digamma_{\phi}=$ int $\bigcap_{i<\alpha} U_{i, \phi(i)}$, then $U\left\{\mathcal{F}_{\phi}: \phi \in \beta^{\alpha}\right\}$ is an almost-cover of $X$ which refines $\mathcal{U}_{i}$ for each $i<\alpha$. This completes the proof that (a) implies (b).

Assume that (b) holds. Suppose that $f, f_{i, j} \in C(X)+(i<\alpha, j<\beta)$ satisfy $0<f=\Sigma_{j<\beta} f_{i, j}$ for all $i<\alpha$. Let $r$ be a positive real number such that $f(y)=r$ for some $y$ in $X$. Consider the cozerosets

$$
U_{i, j}=\left\{x \in X: f(x)<f_{i, j}(x)+r / 3\right\} .
$$

Since $\Sigma_{j<\beta} f_{i, j}=f$, it follows that $U_{j<\beta} U_{i, j}$ is dense in $X$ for each $i<\alpha$. In other words, $\left\{U_{i, j}: j<\beta\right\}$ is an almost- $\beta$-cover for each $i<\alpha$. The hypothesis implies that $U_{\phi \in \beta^{\alpha}}$ int $\bigcap_{i<\alpha} U_{i, \phi(i)}$ is dense. Let $W=\{x: r / 2<f(x)\}$. There exists $\phi \in \beta^{\alpha}$ such that $V=W \cap$ int $\bigcap_{i<\alpha} U_{i, \phi(i)} \neq \varnothing$. For $x \in V, r / 6=r / 2-r / 3$ $<f(x)-r / 3<f_{i, \phi(i)}(x)$ for all $i<\alpha$. Hence $\Pi_{i<\alpha} f_{i, \phi(i)}=0$ is false. By Theorem 2.6, $C(X)$ is $(\alpha, \beta)$-distributive.

It should be remarked that condition (b) of Theorem 3.5 can be weakened as follows. Define a $\beta$-cover of $X$ to be an almost- $\beta$-cover whose union is $X$. Now (b) holds if and only if whenever $\varkappa_{i}$ is a $\beta$-cover for $i<\alpha$, there exists an almost-cover which refines each $u_{i}$. (We will neither prove nor use this condition.) The one point compactification of the integers may be used as an example to show that almost-cover in the previous sentence cannot be replaced by cover.

3.6. Theorem. $C(X)$ is $(\alpha, \alpha)$-distributive if and only if it is $(\alpha, 2)$-distributive.

Proof. Suppose that $C(X)$ is $(\alpha, 2)$-distributive. We may assume that $\alpha$ is infinite. For each $i<\alpha$, let $\left\{U_{i, j}: j<\alpha\right\}$ be an almost- $\alpha$-cover of $X$. For each pair $i, j<\alpha$, there exists a sequence of cozerosets $\left\{U_{i, j, k}: k<\omega_{0}\right\}$ such that $\bigcap_{k<\omega_{0}} U_{i, j, k}=X-U_{i, j}$. Let $V_{i, j, k, 0}=U_{i, j}$, and let $V_{i, j, k, 1}=U_{i, j, k}$. There exists, by hypothesis, an almost-cover $W$ which refines the almost-2-cover $\left\{V_{i, j, k, r}: r<2\right\}$ for each triplet $i<\alpha, j<\alpha, k<\omega_{0}$. As in the proof of 3.5 it can be shown that $W$ refines each almost- $\alpha$-cover $\left\{U_{i, j}: j<\alpha\right\}$. Thus $C(X)$ is $(\alpha, \alpha)$-distributive. The converse is obvious. 
3.7. Corollary. Let $A$ be a $\Phi$-algebra. Then $A$ is $(\alpha, \alpha)$-distributive if and only if it is $(\alpha, 2)$-distributive.

Proof. Theorems 2.11 and 3.6.

The following proposition of Pierce [8], Smith, and Tarski [14] suggested Theorem 3.6.

3.8. Proposition. Each ( $\alpha, 2)$-distributive Boolean algebra is $(\alpha, \alpha)$ distributive.

3.9. Theorem. Let $X$ be a compact zerodimensional space. Then $C(X)$ is $(\alpha, \beta)$-distributive if and only if the Boolean algebra $B(X)$ of open and closed subsets of $X$ is $(\alpha, \beta)$-distributive.

Proof. By 3.6 and 3.8, it suffices to assume that $\alpha$ and $\beta$ are both infinite.

The lattice of idempotents of $C(X)$ is a regular sublattice which is lattice isomorphic to $B(X)$. By $2.2(\mathrm{a}), B(X)$ is $(\alpha, \beta)$-distributive whenever $C(X)$ is. Conversely, let $\left\{U_{i, j}: j<\beta\right\}$ be an almost- $\beta$-cover of $X$ for each $i<\alpha$. Each $U_{i, j}$ is an open $F_{\sigma}$; hence, for each pair $i<\alpha, j<\beta$, there exists a sequence $\left\{H_{i, j, k}: k<\omega_{0}\right\}$ of open and closed subsets of $X$ such that $U_{i, j}=U_{k<\omega_{0}} H_{i, j, k}$. Observe that the least upper bound of a family of elements of $B(X)$ is the closure of their union. For each $i<\alpha, \mathrm{cl} U_{j<\beta, k<\omega_{0}} H_{i, j, k}=X$. By Proposition 2.7, there is a family $\mathcal{F} \subset B(X)$ such that $U \mathcal{F}$ is dense in $X$ and $\mathcal{F}$ refines $\left\{H_{i, j, k}: j<\beta, k<\omega_{0}\right\}$ for each $i<\alpha$. Then $\mathcal{F}$ is an almost-cover which refines each of the given almost- $\beta$-covers.

3.10. Corollary. If $\beta X$ is zerodimensional, then $C(X)$ is $(\alpha, \beta)$-distributive if and only if $B(X)$ is $(\alpha, \beta)$-distributive.

Proof. Observe that $B(X)$ (resp. $\left.C^{*}(X)\right)$ is isomorphic to $B(\beta X)$ (resp. $C(\beta X))$.

The hypothesis of 3.10 is called for by the fact that there exist zerodimensional spaces whose Stone- Cech compactifications are not zerodimensional $[2 ; 7]$. We do not known if the hypothesis can be replaced by the weaker requirement that $X$ be zerodimensional.

\section{Examples.}

4.1. Proposition. Let $\alpha$ be an infinite cardinal. If $X$ has a dense set $P$ of points with the property that for each $p$ in $P$ the intersection of any $\alpha$-sequence of neighborhoods of $p$ has nonempty interior, then $C(X)$ is $(\alpha, \alpha)$-distributive.

Proof. Let $\left\{\left\{U_{i, j}: j<2\right\}: i<\alpha\right\}$ be an $\alpha$-sequence of almost-2-covers of $X$. Let $V$ be a nonempty open subset of $X$, and pick a point $p \in P \cap V$. Since $X-\left(U_{i, 0} \cup U_{i, 1}\right)$ is a $G_{\delta}$ set with empty interior for each $i<\alpha$, there exists $p(k)<\alpha$ such that $p \in U_{i, p(i)}$. By hypothesis, $\varnothing \neq$ int $\bigcap_{i<\alpha}\left(V \cap U_{i, p(i)}\right)$ Cint $\bigcap_{i<\alpha} U_{i, p(i)}$. Thus $U_{\phi \in 2^{\alpha}}$ int $\bigcap_{i<\alpha} U_{i, \phi(i)}$ is dense in $X$. By Theorems 3.5 and 3.6, $C(X)$ is $(\alpha, \alpha)$-distributive. 
There are a number of spaces which satisfy the hypothesis of 4.1. A space with a dense set of isolated points has this property for every cardinal $\alpha$. The spaces $\beta N-N$ and $\beta R^{+}-R^{+}$have no isolated points and satisfy this property for $\alpha=\omega_{0}[10]$. ( $R^{+}$is the space of nonnegative real numbers.) Moreover, $\beta R^{+}-R^{+}$is connected. This suggests the problem: given an infinite cardinal $\alpha$, does there exist a connected space $X$ such that $C(X)$ is $(\alpha, \alpha)$ distributive? The answer is yes. Hausdorff $[5$, p. 182] has guaranteed the existence of a totally ordered set $T$ which is dense-in-itself and satisfies: if $\left\{a_{i}: i<\alpha\right\}$ and $\left\{b_{i}: i<\alpha\right\}$ are subsets of $T$ such that $a_{i}<b_{j}$ for all $i, j<\alpha$, then there exists $c$ in $T$ such that $a_{i}<c<b_{j}$ for all $i, j<\alpha$. Let $X$ be the Dedekind completion of $T$ provided with the order topology. Then $X$ is connected and satisfies the hypothesis of 4.1 .

This proposition is related, as most of these results are, to a theorem concerning Boolean algebras. A field of sets is called $\alpha$-complete-in-the-wider sense if, whenever $\Sigma_{i<\alpha} a_{i}$ exists, $\Sigma_{i<\alpha} a_{i}=\bigcup_{i<\alpha} a_{i}$. Certainly every Boolean algebra which is isomorphic to a field of sets which is $\alpha$-complete-in-the-wider sense is $(\alpha, \alpha)$-distributive. Sikorski has proved [12] that a Boolean algebra is isomorphic to such a field of sets precisely when its space of dual prime ideals satisfies the hypothesis of 4.1 .

4.2. Proposition. Suppose that $X$ has an open subspace $U$ with the property: $U$ has a dense subset $\left\{d_{i}: i<\alpha\right\}$ of nonisolated points such that, for each $i<\alpha, d_{i}$ has a neighborhood basis of cardinality not exceeding $\beta$. Then $C(X)$ is not $(\alpha, \beta)$-distributive.

Proof. By Proposition 3.1, we need only show that $C(U)$ is not $(\alpha, \beta)$ distributive. Let $\left\{U_{i, j}: j<\beta\right\}$ be a family of cozerosets of $U$ which form a base for the neighborhoods of $d_{i}$. For $i<\alpha, j<\beta$, pick a cozeroset $V_{i, j}$ of $U$ such that $d_{i} \notin V_{i, j}$ while $V_{i, j} \cup U_{i, j}=U$. Since $d_{i}$ is not isolated, $\left\{V_{i, j}: j<\beta\right\}$ is an almost- $\beta$-cover of $U$ for each $i<\alpha$. However, if the open set $V$ is contained in $\bigcap_{i<\alpha} V_{i, \phi(i)}$ for some $\phi \in \beta^{\alpha}$, then $V \cap\left\{d_{i}: i<\alpha\right\}=\varnothing$. Hence $V=\varnothing$. By 3.5, $C(U)$ is not $(\alpha, \beta)$-distributive.

4.3. Corollary. Let $X$ be a space with a countable base. Then $C(X)$ is $\left(\omega_{0}, 2\right)$-distributive if and only if $X$ has a dense set of isolated points.

4.4. Corollary. $C(X)$ is $(\alpha, \alpha)$-distributive for every cardinal $\alpha$ if and only if $X$ has a dense set of isolated points.

4.5. Corollary. If $X$ is a space without isolated points, such that $X$ has a dense subset of cardinality at most $\alpha$, then $C(X)$ is not $\left(\alpha, 2^{\alpha}\right)$-distributive.

A Boolean analogue to 4.4 is found in Tarski's theorem: a Boolean algebra is atomistic if and only if it is $(\alpha, \alpha)$-distributive for every cardinal $\alpha$ [14]. (Corollary 4.5 was pointed out by the referee.) 
4.6. Proposition. If $C(X)$ is $(\alpha, \beta)$-distributive and $t$ is a $\gamma$-complete map of $X$ onto $Y$, where $\gamma=\max (\alpha, \beta)$, then $C(Y)$ is $(\alpha, \beta)$-distributive.

Proof. By $1.4, t$ induces a $\gamma$-complete isomorphism of $C(Y)$ into $C(X)$. Let $f, f_{i, j} \in C(Y)(i<\alpha, j<\beta)$ be such that $\Sigma_{j<\beta} f_{i, j}=f>0$ for each $i<\alpha$ and $\Pi_{i<\alpha} f_{i, \phi(i)}=0$ for each $\phi \in \beta^{\alpha}$. Then $\Sigma_{j<\beta} \bar{t}\left(f_{i, j}\right)=\bar{t}(f)>0$ for each $i<\alpha$, although $\Pi_{i<\alpha} \bar{t}\left(f_{i, \phi(i)}\right)=0$ for each $\phi \in \beta^{\alpha}$.

4.7. Corollary. (a) If $X$ is the topological product of spaces $X_{i}, i \in I$, and $C(X)$ is $(\alpha, \beta)$-distributive, then each $C\left(X_{i}\right)$ is $(\alpha, \beta)$-distributive.

(b) If $X$ is homeomorphic to an open subspace of a product of real lines, then $C(X)$ is not $\left(\omega_{0}, 2\right)$-distributive.

We say that a function $f$ on $X$ is locally constant on the subset $T$ of $X$ if each point of $T$ has a neighborhood on which $f$ is constant.

4.8. THEOREM. If $C(X)$ is $\left(\omega_{0}, \omega_{0}\right)$-distributive, then every continuous function from $X$ to a separable metric space is locally constant on a dense set.

Proof. Let $f$ be a continuous function from $X$ to the metric space $T$ with countable dense set $S$. For $q$ in $S$ and $n$ in $N$ (the set of positive integers), let

$$
U_{n, q}=\{x \in X: d(f(x), q)<1 / n\} .
$$

For each $n \in N, \bigcup_{q \in S} U_{n, q}=X$. Since each $U_{n, q}$ is a cozeroset, Theorem 3.5 says that $U_{\phi \in S^{N}}$ int $\bigcap_{n \in N} U_{n, \phi(n)}$ is dense in $X$. Moreover, for each $x \in \bigcap_{n \in N} U_{n, \phi(n)},\{\phi(n)\}$ converges to $f(x)$. Thus int $\bigcap_{n \in N} U_{n, \phi(n)}$ is a set of constancy of $f$.

Theorem 4.8 was first formulated in [8] by Pierce in case $X$ is compact and $C(X)$ is $\omega_{0}$-complete. These hypotheses play a strong role in Pierce's proof. Pierce also proved the converse to this theorem under the same restrictions, but for this case his proof will work provided $\beta X$ is zerodimensional. If it could be shown that this hypothesis is not necessary, we would have an interesting characterization of $\left(\omega_{0}, \omega_{0}\right)$-distributivity.

\section{REFERENCES}

1. G. Birkhoff, Lattice theory, Amer. Math. Soc. Colloq. Pub., vol. 25, rev. ed., Amer. Math. Soc., New York, 1948.

2. C. H. Dowker, Local dimension of normal spaces, Quart. J. Math. Oxford Ser. 6 (1955), 101-120.

3. P. Erdös and A. Tarski, On families of mutually exclusive sets, Ann. of Math. 44 (1943), 315-329.

4. L. Gillman and M. Jerison, Rings of continuous functions, Van Nostrand, Princeton, N. J., 1960.

5. F. Hausdorff, Grundzïge der Mengenlehre, Chelsea, New York, 1949.

6. M. Henriksen and D. G. Johnson, On the structure of a class of archimedean lattice-ordered algebras, Fund. Math. 50 (1961), 73-94.

7. J. R. Isbell, Zerodimensional spaces, Tôhoku Math. J. 7 (1955), 1-8.

8. R. S. Pierce, Distributivity in Boolean algebras, Pacific J. Math. 7 (1957), 983-992. 
9. - Distributivity and the normal completion of Boolean algebras, Pacific J. Math. 8 (1958), 133-140.

10. W. Rudin, Homogeneity problems in the theory of Čech-compactifications, Duke Math. J. 23 (1956), 409-419.

11. D. Scott, The independence of certain distributive laws in Boolean algebras, Trans. Amer. Math. Soc. 84 (1957), 258-261.

12. R. Sikorski, Boolean algebras, Springer-Verlag, Berlin, 1960.

13. E. C. Smith Jr., A distributivity condition for Boolean algebras, Ann. of Math. 64 (1956), 551-562.

14. E. C. Smith Jr. and A. Tarski, Higher degrees of distributivity and completeness in Boolean algebras, Trans. Amer. Math. Soc. 84 (1957), 230-257.

UNIVERSITY OF ILLINOIS,

URBANA, ILLINOIS 